\title{
Threshold for Potential Sputtering of LiF
}

\author{
G. Hayderer, M. Schmid, P. Varga, H P. Winter, and F. Aumayr* \\ Institut für Allgemeine Physik, TU Wien, Wiedner Hauptstrasse 8-10, A-1040 Vienna, Austria \\ L. Wirtz, C. Lemell, and J. Burgdörfer \\ Institute for Theoretical Physics, TU Wien, Wiedner Hauptstrasse 8-10, A-1040 Vienna, Austria \\ L. Hägg ${ }^{1}$ and C. O. Reinhold ${ }^{2}$ \\ ${ }^{1}$ Department of Physics and Mathematics, Mid Sweden University, S-851 70 Sundsvall, Sweden \\ ${ }^{2}$ Oak Ridge National Laboratory, Oak Ridge, Tennessee 37831-6373
}

(Received 18 June 1999)

\begin{abstract}
We have measured total sputtering yields for impact of slow $(\leq 100 \mathrm{eV})$ singly and doubly charged ions on LiF. The minimum potential energy necessary to induce potential sputtering (PS) from LiF was determined to be about $10 \mathrm{eV}$. This threshold coincides with the energy necessary to produce a cold hole in the valence band of LiF by resonant neutralization. This allows the first unambiguous identification of PS induced by cold holes. Further stepwise increase of the sputtering yield with higher projectile potential energies provides evidence for additional defect-mediated sputtering mechanisms operative in alkali halides.

PACS numbers: 79.20.Rf
\end{abstract}

In recent studies on the impact of slow multiply charged ions on insulator surfaces, a dramatic increase of the yields for sputtering [1-4] and secondary ion emission [5-8] with projectile charge state has been observed for certain target species as, e.g., $\mathrm{LiF}$ and $\mathrm{SiO}_{2}$. In contrast to the well established process of kinetically induced sputtering, ablation of target atoms and ions due to the potential energy of the projectile, henceforth called potential sputtering (PS), is largely unexplored. PS can result in high sputter yields at low impact energy and, unlike kinetically induced sputtering, is not accompanied by strong radiation defects in deeper target layers. It has therefore the potential of acquiring considerable technological relevance: Preferential removal of insulating layers (PS is absent for conducting surfaces) could be the basis for novel cleaning procedures for semiconductors (e.g., soft sputtering of $\mathrm{SiO}_{2}$ from $\mathrm{Si}$ wafers). Other applications such as nanostructuring and controlled surface modifications of insulators are also conceivable. A detailed understanding of mechanisms responsible for the conversion of projectile potential energy in PS processes is therefore highly desirable.

Presently, several complementary models for different surface materials are being considered to explain PS. For impact of ions in very high charge states $q$ (up to $q=70$ for $\mathrm{Th}$ ) on uranium oxide, it has been speculated [3] that a "Coulomb explosion" mechanism $[9,10]$ is responsible for the observed strong increase of ablation and secondary ion yields with $q$. For comparably highly charged ions on GaAs, a model involving structural instabilities arising from the destabilization of atomic bonds due to a high density of electronic excitations [11] was invoked to explain the observed high sputtering yields [4]. For projectile ions in somewhat lower charge states $(q \leq 27)$, a large amount of experimental data for various target surfaces (among them alkali halides and $\mathrm{SiO}_{2}$ ) $[1,2,12]$ are at variance with the Coulomb explosion mechanism [13]. They are, however, consistent with the so-called "defect-mediated desorption" model originally developed for electron- and photon-stimulated desorption [14] for alkali halides. In this model, localized defects (e.g., "self-trapped excitons," STE) are formed following particle-hole excitations in the valence band of insulators with strong electron-phonon coupling such as alkali halides and silicon dioxide. Desorption of mostly neutral atoms follows the diffusion of defects to the surface. Theoretical estimates [13] show that the localization of electronic excitations by forming defects is an essential precursor for sputtering. Otherwise, the lifetime of vacancies in the valence band is too short even in insulators for Coulomb explosion to contribute significantly to sputtering (the only exception being sputtering of protons by multiply charged ions $[7,15])$.

Formation of a self-trapped exciton requires a threshold excitation energy (20 eV for LiF, cf. Fig. 1) which can be delivered by the slow incident ion primarily by Auger capture. However, since resonant capture (Fig. 1 in distant ion-surface collisions is much more likely than Auger capture, holes rather than particle-hole excitations are the dominant electronic defects created by slow highly charged particle impact. It was therefore speculated [13] that cold hole production ("self-trapped hole," STH) by capture may play a significant role as precursor for potential sputtering. Such a mechanism would be unique to ion impact as it should be absent in photon- or electronstimulated desorption.

In order to identify specific sputtering mechanisms, we searched for the potential energy threshold, i.e., the minimum potential energy necessary to induce PS. These 


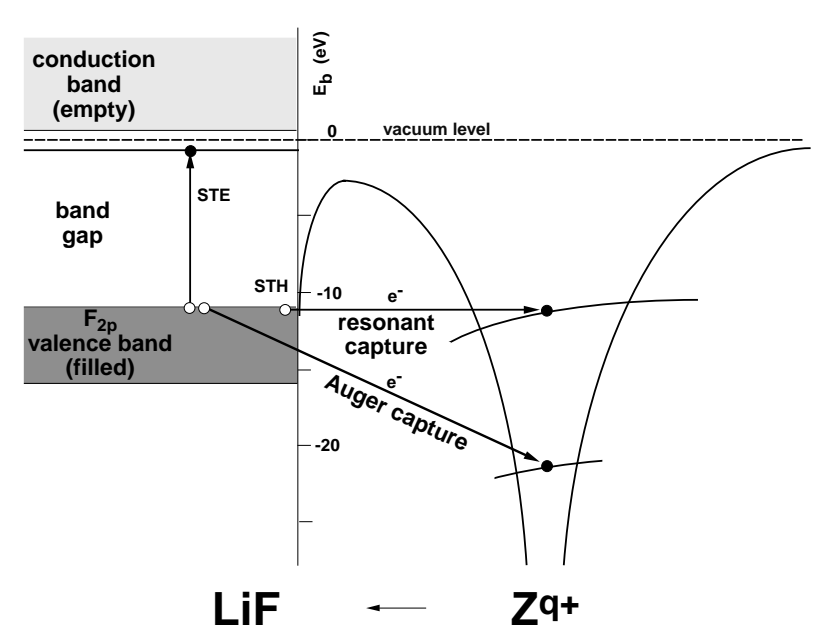

FIG. 1. Formation of holes and electron-hole pairs by resonant- and Auger-capture processes during the interaction of singly or multiply charged ions with a LiF surface (schematically).

investigations were performed for $\mathrm{LiF}$, a prototype wide band gap insulator. By using various singly and doubly charged ions the incident potential energy could be varied from $5.1 \mathrm{eV}\left(\mathrm{Na}^{+}\right)$to $62.6 \mathrm{eV}\left(\mathrm{Ne}^{2+}\right)$. Ions were extracted from a $5 \mathrm{GHz}$ electron cyclotron resonance ion source [16] equipped with an oven to produce ions from metals in addition to the standard ions produced from gases. Kinetic sputtering was suppressed or at least kept at a minimum by decelerating the projectiles to impact energies as low as $20 \mathrm{eV}$. Our experimental technique has been described in detail elsewhere $[1,2,12,13,17]$. In short, total sputter yields (including both neutral and charged secondary particles) for ion-surface collisions are determined with a sensitive quartz-crystal microbalance technique. The target material (in this case LiF) is first deposited on the quartz crystal as a thin polycrystalline film and then bombarded by slow singly or multiply charged ions. The sputter yield is determined from the frequency change of the quartz crystal induced by the mass loss of the target film. Since in the threshold region we are confronted with very small sputtering yields, we had to increase the sensitivity of our quartz-crystal microbalance technique considerably. Using a newly designed electronics, we are now able to detect mass changes as small as $0.5 \%$ of a monolayer per minute [17], which is an improvement by more than a factor of 5 compared to our previous sensitivity limit.

In Fig. 2 the mass change (in atomic mass units per incident ion) is plotted as a function of the potential energy $E_{\text {pot }}$ (ion recombination energy) carried into the collision by the singly and the doubly charged ions. Open and closed symbols correspond to the cases of 20 and $100 \mathrm{eV}$ kinetic impact energy, respectively. The total sputter yield (ordinate on the right-hand side) is obtained by assuming stoichiometric sputtering ( $\sim 26 \mathrm{amu}$ corresponds to one $\mathrm{LiF}$ molecule). For $\mathrm{Na}^{+}, \mathrm{Cu}^{+}$, and $\mathrm{Zn}^{+}$projectiles, all of which carry a potential energy less than $10 \mathrm{eV}$, no

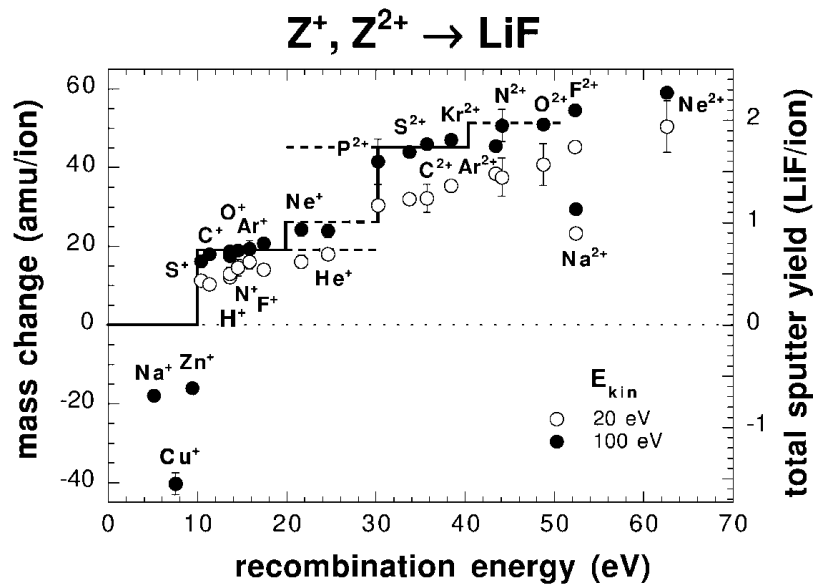

FIG. 2. Mass change due to sputtering (in atomic mass units per incident ion) as a function of the potential energy $E_{\text {pot }}$ (recombination energy) carried by singly and doubly charged projectile ions. Open and full symbols correspond to 20 and $100 \mathrm{eV}$ kinetic impact energy, respectively. The solid line indicates PS yields as expected from simple considerations (cf. text).

mass decrease is observed. Instead, the quartz-crystal microbalance detects a frequency shift corresponding to material deposition on the LiF surface. From the observed mass increase for $\mathrm{Na}$ and $\mathrm{Cu}$ ions, we determine a sticking coefficient of about 0.7 (assuming that no desorption takes place). For $\mathrm{Zn}^{+}$the apparent sticking coefficient is reduced to 0.25 despite similar masses and comparable ionization energies of $\mathrm{Cu}$ and $\mathrm{Zn}$. $\mathrm{Na}$ and $\mathrm{Zn}$ share a similarly high vapor pressure which would lead to almost immediate desorption of metal overlayers (surface clusters) at temperatures typical for our experiment $\left(\sim 180^{\circ} \mathrm{C}\right)$. Although binding of $\mathrm{Zn}^{+}$ions at fluorine sites is less probable than for $\mathrm{Na}^{+}$this difference is not likely to explain a reduction of the sticking probability by a factor of almost 3. A possible reason for this surprising result could be the interplay between sticking (with coefficients for $\mathrm{Zn}$ ions comparable to those for $\mathrm{Na}$ and $\mathrm{Cu}$ ) and the onset of target desorption by $\mathrm{Zn}^{+}$projectiles.

All other projectiles with ionization potentials larger than that of $\mathrm{Zn}$ sputter $\mathrm{LiF}$ even at only $20 \mathrm{eV}$ impact energy. The sputtering yield is typically $20 \%-30 \%$ higher for $100 \mathrm{eV}$ kinetic energy than for $20 \mathrm{eV}$. We explain this residual kinetic energy dependence by a small additional energy transfer from the projectile to the target which facilitates the knock off of loosely bound $\mathrm{F}^{0}$ from the surface. A clear threshold for PS between $9.4 \mathrm{eV}\left(\mathrm{Zn}^{+}\right)$ and $10.4 \mathrm{eV}\left(\mathrm{S}^{+}\right)$is evident. This threshold roughly coincides with the energy to produce one STH via resonant capture (cf. Fig. 1) which leads to the emission of a $\mathrm{F}^{0}$ atom (solid line in Fig. 2). Above $10.4 \mathrm{eV}$ the sputtering yield increases weakly with increasing $E_{\text {pot }}$. As soon as sputtering becomes prevalent, sticking does not have to be taken into consideration in the data evaluation since after a few seconds of ion bombardment an equilibrium between deposition and sputtering of primary particles is reached. 
At a potential energy of about $20 \mathrm{eV}$ the threshold for Auger capture is reached (see Fig. 2), where one electron from the valence band is transferred to an ionic level and the excess energy is used to excite another electron to an excitonic level, thereby leading to the creation of a STE and a STH. The STE can cause the removal of one $\mathrm{F}^{0}$ and one $\mathrm{Li}^{0}$ atom from the surface via decay into an $\mathrm{H}$ and $\mathrm{F}$ center in addition to the removal of one $\mathrm{F}^{0}$ atom by decay of the $\mathrm{STH}$. Mass removal due to the decay of either a STH, a STE, or a combination of STH and STE is indicated in Fig. 2 by dashed lines. The observed mass removal depends on the branching ratio between these decay channels. In our data a distinct second step is hardly recognizable, possibly suggesting that the conversion efficiency of STE's is relatively small. When changing from singly to doubly charged projectiles, the stepwise neutralization $\left(Z^{2+} \rightarrow Z^{+} \rightarrow Z^{0}\right)$ leads to the formation of at least one additional STH if $10 \mathrm{eV}$ potential energy remains for the last neutralization step $\left(Z^{+} \rightarrow Z^{0}\right)$. This is the case for all our doubly charged ions with the exception of $\mathrm{Na}^{2+}$, and, consequently, a considerable increase in the sputtering yield can be observed at around $30 \mathrm{eV}$. Although $\mathrm{Na}^{2+}$ ions carry more than $50 \mathrm{eV}$ potential energy, almost all of this energy is used in the first neutralization step $\left(\mathrm{Na}^{2+} \rightarrow \mathrm{Na}^{+}\right)$. Because of the relatively high kinetic energy of the Auger electron $(\sim 20 \mathrm{eV})$ the induced electronic defect is not likely to localize as a STE but instead as a STH, and the remaining potential energy of $5.1 \mathrm{eV}$ for $\mathrm{Na}^{+} \rightarrow \mathrm{Na}^{0}$ is below the threshold for PS, as shown for $\mathrm{Na}^{+}$projectiles. The exceptionally small sputtering yield for $\mathrm{Na}^{2+}$ (when compared to other doubly charged ions) is therefore fully consistent with the model of sputtering mediated by an ensemble of electronic defects (STH, STE) created by the charge transfer to the projectile (resonant capture, Auger capture). In turn, the available potential energy determines the number and type of defect-generating neutralization processes.

All measurements have been conducted also with projectile ions at higher kinetic energies (500 and $1000 \mathrm{eV}$ ). Transfer of the projectile's kinetic energy to target atoms leads to a considerable increase in the sputtering yields (also for $\mathrm{Na}^{+}, \mathrm{Cu}^{+}$, and $\mathrm{Zn}^{+}$projectiles). A correlation, however, between the projectile's potential energy and the corresponding sputtering yield is no longer observed. For example, $1000 \mathrm{eV} \mathrm{O}^{+}$ions are 3 times more effective in sputtering than $1000 \mathrm{eV} \mathrm{H}^{+}$projectiles, although both carry the same amount of potential energy $(13.6 \mathrm{eV})$.

In order to determine the threshold for defect production (STH) theoretically we performed a simple two-state curve-crossing analysis between the perturbed valence band of $\mathrm{LiF}$ representing the initial channel $\left(\mathrm{F}^{-}+Z^{+}\right)$ and the perturbed ground state of the projectile representing the final channel $(F+Z)$. Because of the high density of states in the initial channel, we assume that charge transfer will occur whenever the diabatic projectile level dives into the shifted valence band level (Fig. 3). The diabatic energy levels in front of a LiF surface were calculated using the Madelung potential and the dielectric response of the surface [18]. At large distances from insulators, the shift of projectile levels is characteristically different from that in front of metals: There, projectile levels are shifted upwards due to the image potential of the ionic core of charge $q$ and the self-image potential of the electron, $\Delta E_{\mathrm{im}}=(q-1 / 2) / 2 z$ [19]. For a singly charged ion in front of an insulator surface, this trend can be reversed for two reasons: Because of the finite dielectric response of the surface $[\chi=(\epsilon-1) /(\epsilon+1)<1, \epsilon<\infty]$, the image potential shift is less pronounced. Furthermore, the electronic state in the exit channel is subject to an effective Madelung potential given by the sum over all Coulomb potentials of the crystal ions. Since capture of an electron leaves a hole at a halide site in the surface, the level shift due to the Madelung potential is negative with an asymptotic behavior of $-(1-\chi) / R$ for $R \rightarrow \infty$, with $R$ being the distance between the electron and the halide hole. The second term in the numerator describes the partial screening of the hole by the dielectric response of the surface [18]. It should be noted that, without the energy shifts of the projectile and target levels, the minimum potential energy for an ion to allow resonant electron capture from the surface would be about $12 \mathrm{eV}$ corresponding to the work function of $\operatorname{LiF}[20,21]$. When the ion comes close to the surface $(R \simeq d)$, the asymptotic description of the level shift $(R \rightarrow \infty)$ breaks down and we smoothly extrapolate the "quasimolecular" potential curves to approach the value of the molecular ionization potential when the distance between the projectile $Z$ and the halide reaches the

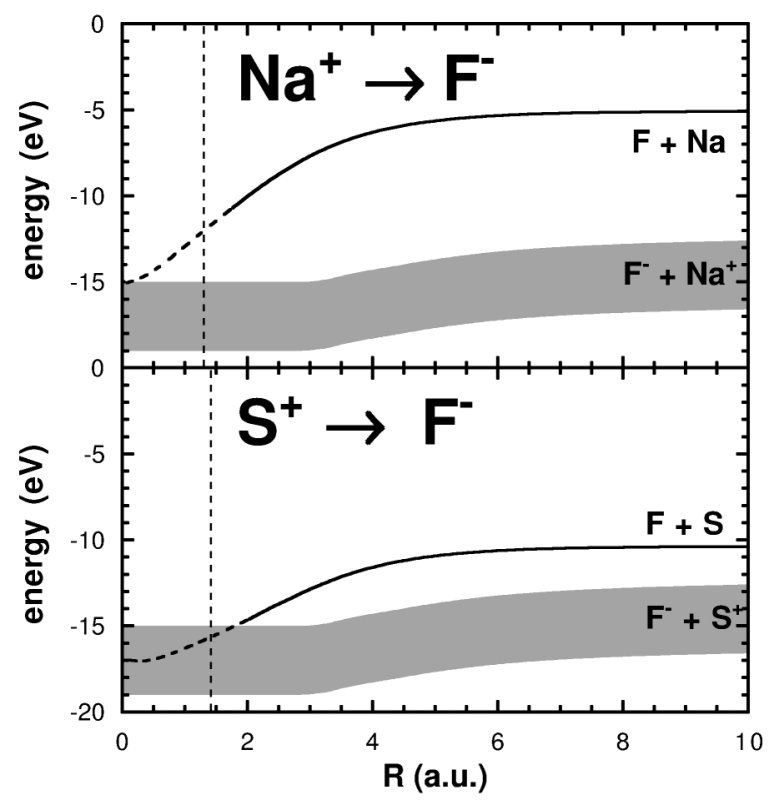

FIG. 3. Potential energy diagrams for $\mathrm{Na}^{+}$and $\mathrm{S}^{+}$as a function of the distance $R$ from the surface (top of $\mathrm{F}^{-}$site). The grey area represents the valence band; the thick solid line shows the projectile energy level. The vertical dashed line indicates the distance of closest approach between projectile and $\mathrm{F}^{-}$. 


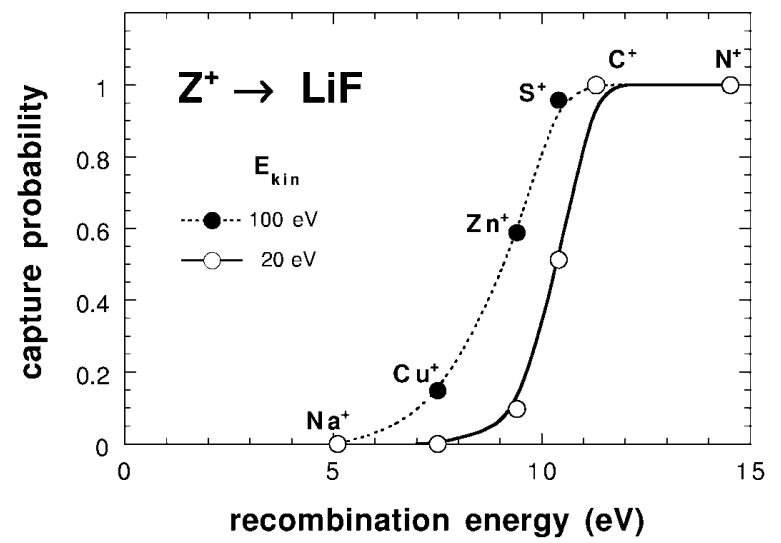

FIG. 4. Calculated capture probability vs ionization potential for different singly charged ions impinging on $\mathrm{LiF}$. Open and full symbols denote results for $20 \mathrm{eV}$ and $100 \mathrm{eV}$ initial kinetic energy, respectively.

bond length $d$ of the isolated molecule and to converge to the united-atom limit as $R \rightarrow 0$. Clearly, a full quantum calculation in terms of molecular orbitals would be desirable (see, e.g., [22]).

Examples for the resulting quasimolecular energy levels for the two-state system (projectile level + valence band) are shown in Fig. 3 for $\mathrm{Na}^{+}$and $\mathrm{S}^{+}$approaching the $\mathrm{LiF}$ surface on top of a $\mathrm{F}^{-}$site. The vertical lines indicate the distance of closest approach of a $100 \mathrm{eV}$ projectile calculated using a Ziegler-Biersack-Littmark potential [23] for the binary encounter collisions with a surface ion. Charge transfer between the valence band and the projectile level can occur only when the crossing with the top of the valence band occurs at distances larger than the turning point. The ground state of the level converging to $\mathrm{Na}$ stays well above the band edge such that resonant neutralization is not possible. Consequently, no hole can be created and hole mediated sputtering should be suppressed. By contrast, for $\mathrm{S}^{+}$the projectile level clearly falls below the band edge before the projectile reaches its classical turning point and STH formation should be possible. Averaging over all impact parameters (different impact sites) in the surface unit cell was performed by Monte Carlo sampling for different ions $\left(\mathrm{Na}^{+}, \mathrm{Cu}^{+}, \mathrm{Zn}^{+}, \mathrm{S}^{+}, \mathrm{C}^{+}, \mathrm{N}^{+}\right)$and normal incidence on the surface. Trajectories with impact energies of 20 and $100 \mathrm{eV}$ were calculated using a molecular dynamics technique.

In agreement with the experimental data (Fig. 2), the fraction of trajectories leading to electron capture from the surface (Fig. 4) as a function of the ionization energy of the ions displays a threshold close to $10 \mathrm{eV}$. While in the case of $20 \mathrm{eV}$ impact energy the threshold is very pronounced, the higher kinetic energy of $100 \mathrm{eV}$ leads to smaller distances of closest approach and therefore to increased capture probability even for projectiles with lower ionization potentials $\left(\sim 15 \%\right.$ for $\left.\mathrm{Cu}^{+}\right)$. A fraction of $50 \% \mathrm{Zn}^{+}$ions capturing an electron is consistent with the estimated balance between sticking and desorption for this projectile. A more detailed account of our simulation will be given in a forthcoming publication.

In summary, our results give convincing evidence that defect-mediated desorption is the dominant mechanism for PS from alkali halides for impact of low charge state ions. We further provide first evidence for the cold-hole mediated sputtering mechanism induced by the resonant charge transfer to the projectile. A threshold of about $10 \mathrm{eV}$ potential energy was found in agreement with a simple two-state curve-crossing model. The resulting defect formed in LiF is a self-trapped hole which can lead to the desorption of a neutral $\mathrm{F}^{0}$ atom or $19 \mathrm{amu}$ per incident ion.

This work has been supported by Austrian Fonds zur Förderung der Wissenschaftlichen Forschung (P12388 and P12470-TH). Participation of S. Cernusca in some of the experiments is gratefully acknowledged.

*Corresponding author.

Email address: aumayr@iap.tuwien.ac.at

[1] M. Sporn et al., Phys. Rev. Lett. 79, 945 (1997).

[2] T. Neidhart et al., Phys. Rev. Lett. 74, 5280 (1995).

[3] T. Schenkel et al., Phys. Rev. Lett. 80, 4325 (1998).

[4] T. Schenkel et al., Phys. Rev. Lett. 81, 2590 (1998).

[5] S. Della-Negra et al., Phys. Rev. Lett. 60, 948 (1988).

[6] G. Schiwietz et al., Radiat. Eff. Defects Solids 127, 11 (1993).

[7] N. Kakutani et al., Nucl. Instrum. Methods Phys. Res., Sect. B 96, 541 (1995).

[8] T. Neidhart et al., Nucl. Instrum. Methods Phys. Res., Sect. B 98, 496 (1995).

[9] I. S. Bitensky and E.S. Parilis, J. Phys. (Paris) C 2, 227 (1989).

[10] H.P. Cheng and J.D. Gillaspy, Phys. Rev. B 55, 2628 (1997).

[11] P. Stampfli, Nucl. Instrum. Methods Phys. Res., Sect. B 107, 138 (1996).

[12] P. Varga et al., Phys. Scr. T73, 307 (1997).

[13] F. Aumayr et al., Comments At. Mol. Phys. 34, 201 (1999).

[14] M. Szymonski, Mat. Fys. Medd. K. Dan.Vidensk. Selsk. 43, 495 (1993), and references therein.

[15] J. Burgdörfer and Y. Yamazaki, Phys. Rev. A 54, 4140 (1996).

[16] M. Leitner et al., Rev. Sci. Instrum. 65, 1091 (1994).

[17] G. Hayderer et al., Rev. Sci. Instrum. 70, 3696 (1999).

[18] L. Hägg et al., Phys. Rev. A 55, 2097 (1997).

[19] J. Burgdörfer et al., Phys. Rev. A 44, 5674 (1991); C. Lemell et al., Phys. Rev. A 53, 880 (1996).

[20] W. Pong and C. S. Inouye, J. Electron Spectrosc. Relat. Phenom. 11, 165 (1977).

[21] M. Piacentini and J. Anderegg, Solid State Commun. 38, 191 (1981).

[22] S. Deutscher et al., Phys. Rev. A 59, 4446 (1999).

[23] J.F. Ziegler et al., The Stopping and Range of Ions in Solids (Pergamon, New York, 1985). 